\title{
Ordonnance 2 COVID-19: pas de fermeture obligatoire des cabinets
}

\author{
PHILIPPE GEISSBÜHLER et GRÉGOIRE GEISSBÜHLER
}

Rev Med Suisse 2020; 16: 750-1

\section{INTRODUCTION}

L'ordre est donné en ce 16 mars 2020: il est interdit aux cabinets médicaux de réaliser des examens, des traitements et des thérapies non urgents. Comment peut-on prendre une pareille décision? La nonurgence est définie comme ce qui peut être différé sans préjudice autre que mineur. Parce qu'on le sait d'avance? La vraie urgence est rare dans la pratique ambulatoire parce qu'on s'emploie justement à éviter qu'elle advienne. Faut-il continuer à consulter clandestinement? Sommes-nous punissables si l'on continue à faire ce qui est de notre devoir?

Face à cette nouvelle problématique juridico-médicale, les deux docteurs Geissbühler, père et fils, reprennent du service.

\section{DISPOSITIONS LÉGALES PERTINENTES}

Le texte légal pertinent est l'Ordonnance 2 sur les mesures destinées à lutter contre le coronavirus (COVID-19) du 13 mars 2020, dite «Ordonnance 2 COVID-19». Cette Ordonnance a été adoptée directement par le Conseil fédéral en vertu des pouvoirs qui lui sont conférés par l'art. 7 de la Loi sur les épidémies en cas de «situation extraordinaire» et directement par l'art. 185 al. 3 de la Constitution fédérale en cas de menace grave pour l'ordre public ou la sécurité intérieure.

Dans le contexte qui nous intéresse ici, deux dispositions sont plus particulièrement intéressantes: l'article 6, qui liste quels établissements restent ouverts ou doivent être fermés, et l'article 10a, qui porte spécifiquement sur les établissements de santé. Dans la mesure où ils concernent les cabinets médicaux, ces dispositions méritent d'être citées dans leur intégralité:

Pour l'article 6:

«Les établissements publics sont fermés, notamment: [...] les prestataires offrant des services impliquant un contact physique tels que salons de coiffure, de massage, de tatouage ou de beauté.» (art. 6 al. 2 let.e O2 COVID-19);

"L'al. 2 ne s'applique pas aux établissements et manifestations suivants: [...] établissements de santé tels qu'hôpitaux, cliniques et cabinets médicaux ainsi que cabinets et établissements gérés par des professionnels de la santé au sens du droit fédéral et cantonal; (art. 6 al. 3 let. $m$ O2 COVID-19);

«Les établissements et manifestations visés à l'al. 3 doivent respecter les recommandations de l'Office fédéral de la santé publique (OFSP) en matière d'hygiène et d'éloignement social. Ils doivent limiter en conséquence le nombre de personnes présentes et empêcher les regroupements de personnes.» (art. 6 al. 4 O2 COVID-19).

Pour l'article 1oa:

«Il est interdit aux établissements de santé au sens de l'art. 6, al. 3, let. m, notamment aux hôpitaux, aux cliniques, aux cabinets médicaux et aux cabinets dentaires, de réaliser des examens, des traitements et des thérapies (interventions) non urgents.» (art. 10a al. 2 O2 COVID-19);

«Sont notamment considérées comme non urgentes les interventions: (a) qui peuvent être réalisées à une date ultérieure sans que la personne concernée ne risque de subir d'autres inconvénients que des atteintes ou des troubles physiques et psychiques mineurs, ou (b) qui sont réalisées, principalement ou entièrement, à des fins esthétiques ou pour améliorer les performances ou le bien-être.» (art. $10 a$ al. 2 O2 COVID-19»;

«Les établissements de santé peuvent pratiquer les interventions légales, prescrites pour des raisons de sécurité au travail, sur les personnes exerçant ou prévoyant d'exercer une activité notamment dans les soins, dans la protection de la population et la protection civile, au sein d'autorités et d'organisations de sauvetage ainsi que pour un service public de sécurité et d'ordre.» (art. 10a al. $4 \mathrm{O}_{2}$ COVID-19».

Signe d'une forme de rétropédalage ou d'une nécessité de clarifier la situation, l'alinéa 2 a été adopté le 16 mars, mais les précisions apportées aux alinéas 3 et 4 n'ont été faites que quatre jours plus tard, le 20 mars - une éternité à l'échelle du temps de crise.

\section{ANALYSE}

À sa lecture, l'O2 COVID-19 semble donner des indications contradictoires sur la pratique en cabinet médical: les établissements où un contact physique avec un patient a lieu sont en principe fermés (art. 6 al. 2 O2 COVID-19), mais les cabinets médicaux sont autorisés (art. 6 al. 3 O2 COVID-19), à condition toutefois de respecter les recommandations en matière de l'OFSP (art. 6 al. 4 O2 COVID-19).

De même, les traitements non urgents sont en principe interdits (art. 10a al. $2 \mathrm{O}_{2}$ COVID-19), sauf s'ils sont nécessaires pour la sécurité du travail (art. 10a al. 4 O2 COVID-19). La notion de «non-urgence» est elle-même précisée par l'Ordonnance, mais avec un seuil assez bas: seuls les atteintes mineures et les traitements esthétiques, de performance ou de bienêtre sont concernés (art. 10a al. $3 \mathrm{O}_{2}$ COVID-19).

Pour clarifier ces points, l'OFSP a rendu un Rapport explicatif concernant l'O2 COVID-19 le 25 mars, qu'il a ensuite actualisé. $^{2}$ Il apporte plusieurs informations additionnelles intéressantes.

En premier lieu, il n'est pas question de fermer tous les cabinets médicaux, au contraire:

«Les établissements de santé tels qu’hôpitaux, cliniques, cabinets médicaux, dentaires et vétérinaires doivent poursuivre leur activité. Cela vaut également pour les établissements gérés par des professionnels de la santé au sens du droit fédéral et cantonal.» (Rapport explicatif, p. 10, mise en évidence ajoutée).

En second lieu, la notion d'établissement de santé est large et ne couvre pas uniquement la médecine «facultaire», les autres professions médicales, les professions de la psychologie ou de la santé ainsi que les professions réglementées par le droit cantonal (p.ex. «les acuponcteurs, les opticiens, les hygiénistes dentaires, les guérisseurs-naturopathes, les homéopathes, les podologues, les logopédistes et les thérapeutes en médecine traditionnelle chinoise»), sont aussi visées (Rapport 
explicatif, p. 10).

Toutes ces professions sont soumises aux règles de l'art. 10a O2 COVID-19. Toutefois, on comprend bien que les enjeux de santé publique ne sont pas les mêmes pour les hôpitaux universitaires que pour les homéopathes. L'approche «one-size-fits-all» de l'O2 COVID-19 doit donc être relativisée.

C'est dans ce sens que doivent être comprises les restrictions de l'art. $10 \mathrm{a} \mathrm{O} 2$ COVID-19: le but n'est pas d'arrêter la médecine de ville, mais d'éviter des regroupements de personnes potentiellement malades qui accroîtrait le risque de contamination et - surtout pour les hôpitaux de concentrer les moyens disponibles sur l'épidémie actuelle (Rapport explicatif, p. 17).

Ainsi, les hôpitaux doivent limiter les interventions qui ne sont pas vitales, et les médecins doivent éviter de les favoriser. Le Rapport explicatif donne à cet égard des exemples extrêmes: une injection des lèvres serait interdite, mais les «opérations vasculaires qui, si elles ne sont pas effectuées, entraînent la perte permanente de la fonction d'un membre» seraient autorisées... on pourrait ajouter «évidemment».

Ce sont toutes les interventions «intermédiaires» qui forment une zone grise que l'Ordonnance n'adresse pas. Or, François Pilet faisait déjà remarquer dans un article de $2010^{3}$ que l'on ne pouvait simplement distinguer entre urgences vitales d'un côté et «bobologie» de l'autre. Il existe une myriade de situations intermédiaires, qui ne nécessitent pas une hospitalisation, mais où la présence d'un médecin est nécessaire.

Cette approche doit être reprise ici: ces consultations sont nécessaires pour la population et sans elles, la situation de certains patients pourrait s'aggraver, voire nécessiter une hospitalisation - ce que l'O2 COVID-19 vise précisément à éviter (p.ex.: un traitement mal ajusté, une fracture négligée, une fièvre dissimulant une maladie plus grave, etc.)

Le Rapport explicatif apporte à cet égard deux précisions bienvenues, et qui auraient mérité de figurer dans le texte de l'O2 COVID-19: il n'est souvent pas possible de savoir à l'avance ce qui est urgent ou non, et faute de pouvoir déterminer clairement quelles opérations sont autorisées ou interdites, c'est aux praticiens de terrain plutôt qu'à Messieurs de Berne de déterminer quelles interventions sont urgentes et nécessaires et lesquelles ne le sont pas (Rapport explicatif, p. 18).

\section{CONCLUSIONS}

L'O2 COVID-19 ne fixe pas des règles strictes, pas plus qu'elle n'appelle à la fermeture de tous les cabinets médicaux. D'ailleurs, les mesures de l'art. 10a ne sont pas visées par les dispositions pénales de l'art. $10 \mathrm{O}$ O2 COVID-19. Il s'agit plutôt d'un guide pour les intervenants du monde de la santé.

La loi ne s'interprète pas uniquement selon son texte ou les explications qu'en donnent le législateur ou ses affidés: elle s'interprète également selon son but (interprétation dite «téléologique»). Quel est le but de cette Ordonnance pour les professions médicales? Elle vise à protéger la santé de la population. Les cabinets médicaux n'ont pas les ressources pour traiter les patients qui nécessitent des soins intensifs, mais ils ont un rôle important pour décharger les hôpitaux et maintenir la population en bonne santé au travers de la crise. Le législateur ferait bien de s'en rappeler.

Cette crise majeure révèle crûment comment les experts et les dirigeants considèrent la médecine ambulatoire en général et la médecine de premier recours en particulier: comme de la camelote dont on peut aisément se passer. Comment interpréter autrement la première réaction du Conseil fédéral et l'interdiction faite aux cabinets médicaux de traiter leurs patients hors urgence, sans plus de précisions? Urgences qui le plus souvent devraient être prises en charge par la médecine hospitalière.

Médecin de premier (et de dernier) recours, le premier auteur de cet article s'est fait au fil du temps une image mentale de sa pratique: une grosse boule de médecine molle englobant un noyau de médecine dure.

La médecine dure est celle que l'on apprend lors de notre cursus hospitalier et universitaire et que l'on remodèle perpétuellement par la formation continue. C'est le domaine de la médecine algorithmique, protocolée, evidence based, scientifique autant que faire se peut, décisionnelle, rapide, puissante, efficace et aussi dangereuse. Celle dont Harari dans Homo deus prétend qu'une intelligence artificielle maîtrise mieux qu'un humain qu'elle pourrait remplacer.

La médecine molle enrobe ce noyau dur d'une couche qui s'épaissit avec le temps. Là se joue l'essentiel: la relation, le temps long, la négociation, le soutien, la discussion, le suivi des traitements, bref la médecine dans un style et un art propre à chacun et qui s'apparente à une sorte de magie relationnelle. Elle permet d'éviter le recours intempestif au noyau dur, les fausses pistes, les effets secondaires. Elle est avant tout thérapeutique, mais il est difficile de chiffrer son efficacité, ce qui la rend vulnérable aux attaques d'experts avides de lean management et de dégraissages, ceux qui nous ont déjà amputés de la liberté de disposer du temps de consultation.

Toutefois, cette efficacité se traduit en creux par l'économie des ressources de la médecine dure qu'elle permet. La médecine d'urgence et hospitalière n'est pas faite pour le suivi des patients. Empêchez la médecine ambulatoire de pratiquer et le système cassera: il sera débordé par des consultations inappropriées pour le système hospitalier de patients laissés à l'abandon. Dans la période actuelle, laissez donc la médecine molle amortir le choc!

\section{IMPLICATIONS PRATIQUES}

- Peut-on, en l'absence de nouvelle contrainte fédérale, continuer à exercer son art au chevet de l'humanité souffrante?

- La réponse est oui

- Mais ne pas oublier de porter un masque

1 Publié au recueil systématique du droit fédéral sous la référence 818.101.24; https:/www.admin.ch/opc/fr/ classified-compilation/20200744/index.html

2 www.bag.admin.ch/dam/bag/fr/dokumente/mt/k-und-i/ aktuelle-ausbrueche-pandemien/2019-nCoV/covid-19-erlaeuterungen-zur-verordnung-2.pdf.download.pdf/ Rapport_explicatif_concernant_I_ordonnance\%202_COVID-19.pdf.

3 Pilet François. Vous avez dit « bobologie » ? Rev Med Suisse 2010;6:736-7.

\section{DR PHILIPPE GEISSBÜHLER}

Spécialiste médecine interne 25 place de la Gare, 2800 Delémont geissbuhler.ph@hin.ch

\section{DR IUR. GRÉGOIRE GEISSBÜHLER}

Avocat (LALIVE, Genève),

Chargé de cours à l'Université de Lausanne gregoire.geissbuhler@unil.ch 